\title{
The Prognostic Value of Central Lymph Node Yield and Ratio in Papillary Thyroid Carcinoma Patients Who Underwent Thyroidectomy with Prophylactic Central Compartment Neck Dissection
}

\author{
Ohjoon Kwon ${ }^{1}$, Sohee Lee ${ }^{1}$ and Ja Seong Bae ${ }^{2}$ \\ Division of Thyroid \& Endocrine Surgery, Department of Surgery, Eunpyeong St. Mary's Hospital, College of Medicine, The \\ Catholic University of Korea ${ }^{1}$, Division of Thyroid \& Endocrine Surgery, Department of Surgery, Seoul St. Mary's Hospital, College \\ of Medicine, The Catholic University of Korea², Seoul, Korea
}

Background and Objectives: The impacts of prophylactic central compartment neck dissection (PCCND) on the prognosis of papillary thyroid cancer (PTC) are controversial. The aim of this study is to evaluate the relationship between nodal factors of PCCND and the prognosis of PTC patients. Materials and Methods: A total of 1754 patients who underwent thyroidectomy with PCCND were retrospectively reviewed. Nodal factor was defined as the number of metastatic lymph node (MLN), lymph node yield (LNY) and lymph node ratio (LNR). In regarding the cutoff of nodal factors, patients were categorized as low/high MLN, LNR and LNY group. The correlation of clinicopathologic characteristics including nodal factors and recurrence free survival (RFS) were anlalyzed. Results: Of these, 1195 patients underwent thyroidectomy with unilateral PCCND and 559 patients underwent total thyroidectomy with bilateral pCCND. During follow-up, 45 (2.57\%) patients showed recurrent disease. Of these, 19 patients underwent bilateral PCCND and 26 cases were unilateral PCCND. Gross extrathyroidal extension (ETE), high MLN and LNR showed statistically significant on RFS in univariate analysis in unilateral PCCND. In multivariate analysis, gross ETE and high LNR were independent risk factor of recurrence in unilateral PCCND. In bilateral PCCND, larger tumor size, minimal ETE, high MLN and LNR were significant correlation with RFS in univariate analysis. However, in multivariate analysis, multiple larger tumor and high LNR showed significant correlation with RFS. LNY was not statistically significant in both unilateral and bilateral PCCND. Conclusion: In regarding nodal factors, high LNR was only independent risk factor to worse RFS in both unilateral and bilateral PCCND in CNO PTC patients.

Key Words: Papillary thyroid carcinoma, Lymph node yield, Lymph node ratio, Metastatic lymph node

\section{Introduction}

Papillary thyroid carcinoma (PTC) is the most common type of thyroid malignancy. ${ }^{1,2)}$ The surgical removal has been accepted as the mainstay of its treat- ment and radical resection includes both complete removal of the diseased thyroid gland and metastatic lymph nodes. ${ }^{3)}$ Regional lymph node metastasis are frequent at initial diagnosis of PTC, the role of therapeutic lymph node dissection is well established in clinical N1 disease. ${ }^{4)}$ Less resection of lymph nodes

Received May 14, 2019 / Revised May 16, 2019 / Accepted May 16, 2019

Correspondence: Ja Seong Bae, MD, PhD, Division of Thyroid \& Endocrine Surgery, Department of Surgery, Seoul St. Mary's Hospital, College of Medicine, The Catholic University of Korea, 222 Banpo-daero, Seocho-gu, Seoul O659l, Korea Tel: 82-2-2228-6088, Fax: 82-2-595-2822, E-mail: drbae@catholic.ac.kr

Copyright (c) 2019, the Korean Thyroid Association. All rights reserved

() This is an open-access article distributed under the terms of the Creative Commons Attribution Non-Commercial License (http://creativecommons.org/licenses/by-nc/4.0/), which permits unrestricted non-commercial use, distribution, and reproduction in any medium, provided the original work is properly cited. 
and higher proportion of metastatic node per dissected lymph node are known risk factor for poorer prognosis in PTC. ${ }^{5-9)}$ However, in clinically NO patients, there are conflicting data about effects of prophylactic lymph node dissection on long-term outcome, especially in central compartment area. ${ }^{4,5,10,11)}$ Moreover, the impacts of lymph node yield (LNY) and lymph node ratio (LNR) of prophylactic central compartment neck dissection (CCND) on the prognosis of PTC still remain controversial. ${ }^{6,8,9,12,13)}$ Therefore, we evaluated the relationship between nodal factor and the recurrence free survival (RFS) of prophylactic CCND in large-scale PTC cohort.

\section{Materials and Methods}

\section{Patients}

A total of 2604 patients underwent thyroid surgery at Seoul St. Mary's Hospital, College of Medicine, the Catholic University of Korea, from 2009 to 2012. The management of thyroid cancer was determined in according to the guidelines issued by the American Thyroid Association. ${ }^{14)}$ In our institution, prophylactic ipsilateral CCND was performed in all thyroid cancer patients and bilateral pCCND was selectively performed in bilateral thyroid cancer and/or in advanced primary tumor. In this study, we excluded non-classical variants such as follicular variant, tall cell variant, or diffuse sclerosing variant from this study and only patients with classical PTC by the final histologic report were enrolled. Additionally, we excluded the patients who underwent the therapeutic lymph node dissection for clinical N1 disease. Patients who were suspected of malignant lymph nodes in the central compartment or lateral neck on the pre-operative examination were also excluded. For analysis of nodal factor, the patients with minimum of one node was examined at final histology were enrolled.

Finally, we retrospectively reviewed 1754 patients of classic PTC who underwent thyroidectomy with pCCND by a single surgeon (Ja seong Bae, MD, PhD). All patients took levothyroxine for thyroid stimulating hormone suppression postoperatively. Radio- active iodine therapy was performed 6 to 8 weeks after total thyroidectomy, based on risk factors according to the ATA guidelines. ${ }^{14)}$ Serum thyroglobulin levels and neck ultrasonography were regularly checked during follow-up. The histologic report was revised according to AJCC $8^{\text {th }}$ edition. The study protocol was approved by our Institutional Review Board (KC19RESI0015).

\section{Nodal Factors}

To focus on the effect of nodal factor on RFS, patients were divided into unilateral pCCND and bilateral pCCND groups. In unilateral pCCND, ipsilateral para-tracheal, peri-thyroidal and pre-tracheal lymph nodes were included, and sometimes, delphian node was included. Bilateral pCCND included pre-tracheal, both para-tracheal and peri-thyroidal node, and delphian node and occasionally para-esophageal lymph node. The LNY is the total number of lymph nodes removed and LNR was defined as a ratio of the number of metastatic lymph node (MLN) to LNY. In regarding the cutoff of nodal factors, patients were categorized as low/high MLN, LNR and LNY group, respectively.

\section{Statistics}

The groups were compared using the $\chi^{2}$ test, the Mann-Whitney $U$ test, the Student's t test, or Fisher's exact test for qualitative or quantitative variables, as appropriate. Receiver operating characteristic curve analysis (ROC) curves and area under curves (AUC) were used to determine the cut-off values of nodal factors including LNY, LNR and MLN. The univariate and multivariate cox regression analysis were used statistically to analyze the factors affecting RFS. Kaplan-Meier survival analysis was used to compare RFS data between different groups. All Data were analyzed with SPSS 18 and $p<0.05$ was considered statistically significant.

\section{Results}

\section{Clinicopathologic Characteristics}

A total of 1754 patients, 1195 patients underwent 
Table 1. Basal characteristics of patients

\begin{tabular}{|c|c|c|c|}
\hline Variables & $\begin{array}{c}\text { Total patients } \\
n=1754\end{array}$ & $\begin{array}{l}\text { Unilateral pCCND } \\
\qquad n=1195\end{array}$ & $\begin{array}{l}\text { Bilateral pCCND } \\
\qquad n=559\end{array}$ \\
\hline Age (yrs, mean, range) & $45.9(16-81)$ & $45.3(16-78)$ & $47.2(18-81)$ \\
\hline Gender (F:M) & 1448:306 & $971: 244$ & $477: 82$ \\
\hline \multicolumn{4}{|l|}{ Thyroidectomy } \\
\hline LTT & 449 (25.6\%) & $449(37.6 \%)$ & $0(0 \%)$ \\
\hline $\mathrm{TT}$ & $1305(74.4 \%)$ & $746(62.4 \%)$ & $559(100 \%)$ \\
\hline \multicolumn{4}{|l|}{ RAl } \\
\hline None & $1155(65.8 \%)$ & $895(74.9 \%)$ & $260(46.5 \%)$ \\
\hline Yes & $599(34.2 \%)$ & $300(25.1 \%)$ & $299(53.5 \%)$ \\
\hline Tumor size $(\mathrm{cm})$ & $0.8 \pm 0.5$ & $0.7 \pm 0.4$ & $1.0 \pm 0.6$ \\
\hline \multicolumn{4}{|l|}{ Extrathyroidal extension } \\
\hline None & $1012(57.7 \%)$ & $753(63.0 \%)$ & $259(46.3 \%)$ \\
\hline Minimal & $695(39.6 \%)$ & $430(36.0 \%)$ & $265(47.4 \%)$ \\
\hline Gross & $47(2.7 \%)$ & $12(1.0 \%)$ & $35(6.3 \%)$ \\
\hline \multicolumn{4}{|l|}{ Multifocality } \\
\hline None & $1222(69.7 \%)$ & $933(78.1 \%)$ & $289(51.7 \%)$ \\
\hline Yes & 532 (30.3\%) & $262(21.9 \%)$ & $270(48.3 \%)$ \\
\hline \multicolumn{4}{|l|}{ Thyroiditis } \\
\hline None & $1331(75.9 \%)$ & $967(80.9 \%)$ & $364(65.1 \%)$ \\
\hline Yes & $423(24.1 \%)$ & $228(19.1 \%)$ & $195(34.9 \%)$ \\
\hline No. of N1a patients & $754(43.0 \%)$ & $410(34.3 \%)$ & $344(61.5 \%)$ \\
\hline No. of metastatic node & $1.3 \pm 2.4(0-20)$ & $0.8 \pm 1.6(0-12)$ & $2.4 \pm 3.2(0-20)$ \\
\hline Lymph node yield & $8.4 \pm 5.8(1-40)$ & $6.4 \pm 4.0(1-31)$ & $12.8 \pm 6.6(1-40)$ \\
\hline Lymph node ratio & $0.16 \pm 0.25(0-1)$ & $0.14 \pm 0.25(0-1)$ & $0.2 \pm 0.24(0-1)$ \\
\hline Recurrence (n) & $45(2.6 \%)$ & $26(2.2 \%)$ & $19(3.4 \%)$ \\
\hline
\end{tabular}

F: female, LTT: less than total thyroidectomy, M: male, TT: Total thyroidectomy, pCCND: prophylactic central compartment neck dissection, RAl: radioactive iodine ablation

thyroidectomy with unilateral pCCND and 559 patients underwent total thyroidectomy with bilateral PCCND (Table 1). The median follow up duration was 81.5 months (range $0-120$ ). Mean age was 45.9 years old and $1325(75.5 \%)$ patients were younger than 55 years at diagnosis. In unilateral pCCND group, 746 (62.4\%) patients underwent total thyroidectomy. In cases of bilateral pCCND group, all patients underwent total thyroidectomy. Mean tumor size was $0.8 \pm 0.5$ $\mathrm{cm}$. A total 754 (43.0\%) patients showed central neck node metastasis, of these, 410 (34.3\%) patients were unilateral pCCND group and $344(61.5 \%)$ patients were bilateral PCCND group. Mean MLN was $1.3 \pm 2.4,0.8 \pm 1.6,2.4 \pm 3.2$ in entire study group, unilateral $\mathrm{DCCND}$ and bilateral $\mathrm{PCCND}$, respectively. The mean LNY was $6.4 \pm 4.0$ in unilateral pCCND group and $12.8 \pm 6.6$ in bilateral pCCND group. In the aspect of LNR, mean LNR of unilateral group was $0.14 \pm 0.25$ and mean LNR of bilateral group was
$0.2 \pm 0.24$. A total 45 patients $(2.57 \%)$ showed recurrent disease during follow-up. Of these, 19 patients underwent bilateral pCCND and 26 cases were unilateral pCCND. The RFS was 18.7 (3-69) months in bilateral pCCND group and 21.8 (4-78) months in unilateral PCCND. In bilateral PCCND group, recurrence was found in the lateral neck nodes except one patient with thyroid bed recurrence. Among the 26 recurrent patients with unilateral pCCND, 19 patients had recurrence on their lateral neck area, three patients had recurrence on their contralateral thyroid lobe. Three patients showed recurrences at contralateral central node area without dissection at initial surgery and only one case showed recurrence in ipsilateral thyroid bed which was dissected at initial surgery.

\section{Nodal Factors}

The cutoff value of nodal factor was described in 
Nodal Factors Impact on PTC with Prophylactic CCND

Table 2. The cut-off values of nodal factors in bilateral and unilateral CCND group

\begin{tabular}{lllccccc}
\hline & Variables & AUC & p value & Cl (95\%) & Cut-off value & Specificity & Sensitivity \\
\hline \multirow{2}{*}{ Unilateral CCND } & LNY & 0.624 & 0.030 & $(0.504-0.744)$ & 5.5 & 0.654 & 0.463 \\
& LNR & 0.683 & 0.001 & $(0.571-0.796)$ & 0.15 & 0.615 & 0.732 \\
\multirow{3}{*}{ Bilateral CCND } & MLN & 0.695 & 0.001 & $(0.579-0.810)$ & 0.5 & 0.654 & 0.664 \\
& $L N Y$ & 0.584 & 0.215 & $(0.434-0.733)$ & 10.5 & 0.632 & 0.396 \\
& $L N R$ & 0.806 & $<0.0001$ & $(0.706-0.907)$ & 0.3 & 0.789 & 0.739 \\
& MLN & 0.7697 & $<0.0001$ & $(0.689-0.904)$ & 4.5 & 0.632 & 0.813 \\
\hline
\end{tabular}

AUC: area under curves, CCND: central compartment neck dissection, $\mathrm{Cl}$ : confidence interval, LNR: lymph node ratio, LNY: lymph node yield, MLN: metastatic lymph nodes number

Table 2. In the unilateral CCND group, these values were LNY 5.5 ( $A \cup C=0.624$, specificity $=0.654$, sensitivity= 0.463), LNR 0.15 ( $A \cup C=0.683$, specificity $=0.615$, sensitivity $=0.732$ ) and MLN 0.5 ( $A \cup C=0.695$, specificity $=0.654$, sensitivity $=0.664$ ), respectively. In bilateral PCCND group, the cutoff value of LNY was 10.5 ( $A \cup C=0.584$, specificity $=0.632$, sensitivity $=0.396$ ), that of $L N R$ was $L N R 0.3$ ( $A \cup C=0.806$, specificity $=0.789$, sensitivity $=0.739$ ) and that of MLN was 4.5 (AUC= 0.770 , specificity $=0.632$, sensitivity $=0.813$ ), respectively. According to these cutoff values, we categorized the groups according to the criteria as low and high group (Table 3). In the unilateral pCCND group, 410 (34.3\%) patients were high MLN group. A total 633 (53.0\%) unilateral pCCND patients were assigned as high LNY and 329 (27.5\%) were grouped as high LNR. In bilateral pCCND group, $113(20.2 \%)$ patients were high MLN group and 338 (60.5\%) patients were high LNY, $156(27.9 \%)$ were assigned in high LNR.

\section{Univariate and Multivariate Cox Regression Analysis in Unilateral CCND Group (Table 4)}

The univariate and multivariate cox regression analysis was performed to examine the factors affecting RFS in the unilateral CCND group. Gross extrathyroidal extension, high MLN and high LNR showed statistically significant correlation on RFS (hazard ratio [HR] 16.695 [4.754-58.628], $p<0.0001,3.587$ [1.599-8.049], $p<0.002$ and 4.207 [1.909-9.272], $p$ $<0.0001$, respectively). However, age, sex, the extent of thyroidectomy, tumor size category and LNY was not statistically significant. In multivariate cox regression, gross extrathyroidal extension (HR 13.056 [3.687-46.238], $p<0.0001$ ) and high LNR (HR 3.979
Table 3. Nodal factors of unilateral and bilateral pCCND groups

\begin{tabular}{|c|c|c|c|}
\hline \multicolumn{2}{|c|}{ Variables } & \multirow{2}{*}{$\begin{array}{c}\text { Category } \\
M L N<1\end{array}$} & \multirow{2}{*}{$\begin{array}{c}\begin{array}{c}\text { Unilateral pCCND } \\
n=1195\end{array} \\
785(65.7 \%)\end{array}$} \\
\hline MLN groups & Low & & \\
\hline & High & $1 \leq \mathrm{MLN}$ & $410(34.3 \%)$ \\
\hline \multirow[t]{2}{*}{ LNY groups } & Low & LNY $<6$ & 562 (47.0\%) \\
\hline & High & $6 \leq \mathrm{LNY}$ & $633(53.0 \%)$ \\
\hline \multirow[t]{2}{*}{ LNR groups } & Low & $L N R<0.15$ & $866(72.5 \%)$ \\
\hline & High & $0.15 \leq L N R$ & 329 (27.5\%) \\
\hline \multicolumn{2}{|c|}{ Variables } & Category & $\begin{array}{c}\text { Bilateral pCCND } \\
\quad n=559\end{array}$ \\
\hline \multirow[t]{2}{*}{ MLN groups } & Low & $M L N<5$ & $446(79.8 \%)$ \\
\hline & High & $5 \leq \mathrm{MLN}$ & $113(20.2 \%)$ \\
\hline \multirow[t]{2}{*}{ LNY groups } & Low & LNY $<11$ & 221 (39.5\%) \\
\hline & High & $11 \leq \mathrm{LNY}$ & 338 (60.5\%) \\
\hline \multirow[t]{2}{*}{ LNR groups } & Low & $L N R<0.3$ & $403(72.1 \%)$ \\
\hline & High & $0.3 \leq \mathrm{LNR}$ & $156(27.9 \%)$ \\
\hline
\end{tabular}

LNR: lymph node ratio, LNY: lymph node yield, MLN: metastatic lymph nodes number, pCCND: prophylactic central compartment neck dissection

[1.785-8.867], $p=0.001)$ were independent risk factor of recurrence.

\section{Univariate and Multivariate Cox Regression Analysis in Bilateral CCND Group (Table 5)}

In univariate cox regression analysis of bilateral pCCND group, larger tumor size, minimal extrathyroidal extension showed statistical correlation on RFS. In regarding nodal factors, high MLN and high LNR were statistically significant factors for RFS in univariate cox regression analysis (HR 3.587 [1.599-8.049], $p=$ 0.002 and HR 10.216 [3.390-30.783], $p<0.0001$ ). However, LNY had no statistically significant effect on RFS. In multivariate analysis, tumor size between $2 \mathrm{~cm}$ and $4 \mathrm{~cm}$ (HR 9.689 [2.722-34.485], $p<0.0001)$ and 
Table 4. Univariate and multivariate cox regression analysis in unilateral pCCND group

\begin{tabular}{|c|c|c|c|c|}
\hline Variables & & $H R$ & $\mathrm{Cl}(95 \%)$ & $\mathrm{p}$ value \\
\hline \multicolumn{5}{|l|}{ Univariate } \\
\hline Age ( $<55$ vs. $\geq 55$ years) & & 0.449 & $(0.135-1.495)$ & 0.192 \\
\hline Sex (female vs. male) & & 1.603 & $(0.674-3.813)$ & 0.286 \\
\hline Thyroidectomy extent (LTT vs. TT) & & 1.093 & $(0.487-2.456)$ & 0.829 \\
\hline \multirow[t]{3}{*}{ Tumor size $(\mathrm{cm})$} & $\leq 1$ & Reference & & \\
\hline & $1<\leq 2$ & 1.525 & $(0.573-4.064)$ & 0.398 \\
\hline & $2<\leq 4$ & 3.321 & $(0.445-24.763)$ & 0.242 \\
\hline \multirow{3}{*}{ Extrathyroidal extension } & None & Reference & & \\
\hline & Minimal & 1.316 & $(0.577-3.002)$ & 0.514 \\
\hline & Gross & 16.695 & $(4.754-58.628)$ & $<0.0001$ \\
\hline Multifocality (single vs. multi) & & 1.554 & $(0.676-3.537)$ & 0.300 \\
\hline Thyroiditis (no vs. yes) & & 0.794 & $(0.274-2.305)$ & 0.672 \\
\hline MLN (low vs. high) & & 3.587 & $(1.599-8.049)$ & 0.002 \\
\hline LNY (low vs. high) & & 1.686 & $(0.752-3.783)$ & 0.205 \\
\hline LNR (low vs. high) & & 4.207 & $(1.909-9.272)$ & $<0.0001$ \\
\hline Post OP RAl (no vs. yes) & & 1.779 & $(0.807-3.925)$ & 0.153 \\
\hline \multicolumn{5}{|l|}{ Multivariate } \\
\hline \multirow[t]{3}{*}{ ETE } & None & Reference & & \\
\hline & Minimal & 1.057 & $(0.459-2.435)$ & 0.896 \\
\hline & Gross & 13.056 & $(3.687-46.238)$ & $<0.0001$ \\
\hline LNR (low vs. high) & & 3.979 & $(1.785-8.867)$ & 0.001 \\
\hline
\end{tabular}

Cl: confidence interval, HR: hazard ratio, LNR: lymph node ratio, LNY: lymph node yield, LTT: less than total thyroidectomy, MLN: metastatic lymph nodes number, pCCND: prophylactic central compartment neck dissection, post OP: postoperative, RAI: radioactive iodine ablation, TT: total thyroidectomy, vs.: versus

multifocal tumor (HR $2.829[1.053-7.601], p=0.039)$ were independent variables. High LNR remains an independent variable among nodal factors in bilateral CCND group (HR 8.192 [2.699-24.862], p<0.0001).

\section{Recurrence Free Survival According to LNR Groups}

In unilateral pCCND group, patients with $L N R<0.15$ showed 109.0 months in RFS and those with LNR $\geq$ 0.15 did 104.5 months in RFS in unilateral pCCND group $(p<0.0001$, Fig. 1A). In bilateral $p C C N D$ group, the estimated RFS of patients with LNR $<0.3$ was 107.1 months and that of patients with LNR $\geq 0.3$ was 98.9 months $(p<0.0001$, Fig. 1B).

\section{Discussion}

PTC usually spreads via lymphatic channels, cervical lymph node metastasis is common. ${ }^{1)}$ Lymph node metastasis at initial diagnosis was up to $40-60 \%$ of all patients in various studies. ${ }^{14-17)}$ Similarly, in this study, a total 754 (43.0\%) patients showed central neck node metastasis. Regional lymph node metastasis in cases with clinical presentation, larger metastatic node than $3 \mathrm{~cm}$ and multiple metastatic lymph node $(N>5)$ was associated with recurrence.) However, routine lymph node dissection was not recommended in all PTC patients due to minimal effect on survival and issues about complication." According to American thyroid Association guidelines, CCND with therapeutic intent in clinically involved central node was recommend and prophylactic nodal dissection only was recommended in advanced primary tumor. ${ }^{4)}$ Nevertheless, lymph nodes in the central compartment were difficult to evaluate accurately in preoperative imaging studies due to thyroid gland and air-filled trachea. ${ }^{18,19)}$ In a similar view, $116(6.6 \%)$ cNO patients (36 [3.0\%] patients in unilateral PCCND and 80 [14.3\%] patients in bilateral pCCND) showed more than 5 metastatic node which were classified ATA intermediate risk group. ${ }^{4)}$

The impacts of PCCND on the prognosis of PTC were controversial. Some researchers suggest 
Nodal Factors Impact on PTC with Prophylactic CCND

Table 5. Univariate and multivariate cox regression analysis in bilateral pCCND group

\begin{tabular}{|c|c|c|c|c|}
\hline Variables & & $H R$ & $\mathrm{Cl}(95 \%)$ & $p$ value \\
\hline \multicolumn{5}{|l|}{ Univariate } \\
\hline Age (<55 vs. $\geq 55$ years) & & 0.445 & $(0.130-1.528)$ & 0.198 \\
\hline Sex (female vs. male) & & 1.541 & $(0.511-4.644)$ & 0.442 \\
\hline \multirow[t]{4}{*}{ Tumor size $(\mathrm{cm})$} & $\leq 1$ & Reference & & \\
\hline & $1<\leq 2$ & 3.896 & $(1.306-11.626)$ & 0.015 \\
\hline & $2<\leq 4$ & 10.880 & $(3.148-37.596)$ & $<0.0001$ \\
\hline & $4<*$ & $<0.0001$ & N/A & 0.989 \\
\hline \multirow[t]{3}{*}{ Extrathyroidal extension } & None & Reference & & \\
\hline & Minimal & 3.253 & $(1.061-9.997)$ & 0.039 \\
\hline & Gross & 3.816 & $(0.699-20.835)$ & 0.122 \\
\hline Multifocality (single vs. multi) & & 2.332 & $(0.887-6.137)$ & 0.086 \\
\hline Thyroiditis (none vs. present) & & 0.854 & $(0.325-2.247)$ & 0.749 \\
\hline MLN (low vs. high) & & 7.086 & $(2.790-18.000)$ & $<0.0001$ \\
\hline LNY (low vs. high) & & 1.128 & $(0.444-2.866)$ & 0.799 \\
\hline LNR (low vs. high) & & 10.216 & $(3.390-30.783)$ & $<0.0001$ \\
\hline Post OP RAl (no vs. yes) & & 1.852 & $(0.704-4.872)$ & 0.212 \\
\hline \multicolumn{5}{|l|}{ Multivariate } \\
\hline \multirow[t]{4}{*}{ Tumor size $(\mathrm{cm})$} & $\leq 1$ & Reference & & \\
\hline & $1<\leq 2$ & 2.985 & $(0.993-8.972)$ & 0.051 \\
\hline & $2<\leq 4$ & 9.689 & $(2.722-34.485)$ & $<0.0001$ \\
\hline & $4<*$ & 0.001 & N/A & 0.989 \\
\hline Multifocality (single vs. multiple cancer) & & 2.829 & $(1.053-7.601)$ & 0.039 \\
\hline LNR (low vs. high) & & 8.192 & $(2.699-24.862)$ & $<0.0001$ \\
\hline
\end{tabular}

*Patient with $4 \mathrm{~cm}<$ tumor size was only one in bilateral CCND group.

Cl: confidence interval, HR: hazard ratio, LNR: Iymph node ratio, LNY: lymph node yield, MLN: metastatic lymph nodes number, N/A: not available, pCCND: prophylactic central compartment neck dissection, post OP: postoperative, RAl: radioactive iodine ablation, vs.: versus

A

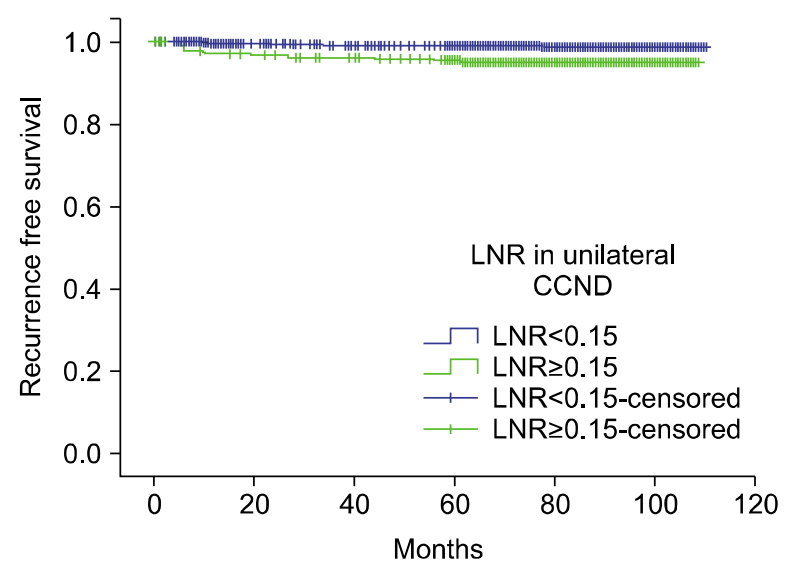

B

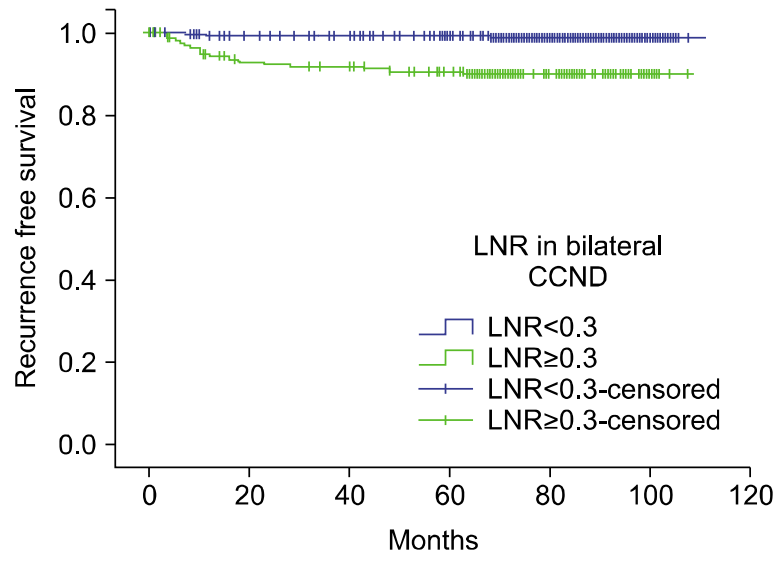

Fig. 1. Difference of recurrence free survival (RFS) according to lymph node ratio in two groups. (A) Kaplan-Meier survival analysis of RFS in bilateral central compartment neck dissection (CCND) group. (B) Kaplan-Meier survival analysis of RFS in unilateral CCND group.

pCCND may convert clinical NO patients to pathologic $\mathrm{N} 1 \mathrm{a}$, and it results upstaging and may lead overtreatment without definite survival benefit., ${ }^{4,14)}$ Other studies demonstrated higher morbidity of pCCND including transient/permanent hypoparathyroidism and recurrent laryngeal injury with no improvement of prognosis. ${ }^{4,14,20)}$ Some researchers reported that pCCND reduced local recurrence. ${ }^{10,11,21,22)}$ Furthermore, prophylactic node 
dissection has been suggested to reduce post-treatment serum Tg levels and to improve disease specific survival. ${ }^{5,10,21)}$ Furthermore, preoperative evaluation of According to guideline of Japanese Association of Endocrine/Japanese Society of Thyroid Surgery, pCCND was generally performed due to it reduced recurrence rate on central compartment area and prevented the inevitable increase in complication from secondary surgery. ${ }^{22)}$ In this study, only 45 cases (2.57\%) showed recurrence among 1754 patients and the recurrence rate was very low. Additionally, most recurrences were found in the lateral neck area of the lesion side. Only five had recurrence at the central compartment area, of these, only two patient showed recurrences in which was already dissected at initial surgery.

In regarding nodal factors, various variables were considered. Numbers of metastatic lymph node were known to be a significant risk factor for recurrences. $^{23-27)}$ Current staging system is divided nodal status into a binary form (NO vs. N1), however, revised ATA guideline reflected the number and size of metastatic nodes at initial risk stratification. ${ }^{4)}$ Park et al. ${ }^{24)}$ reported the cutoff of MLN as 3 in total thyroidectomy and bilateral CCND in N1a patients and Randolph et $\mathrm{al}^{28)}$ reported the cutoff of $\geq 5$ in based on various previous reports. In this study, we used ROC curve to determine the cutoff of MLN which could predict recurrence best in unilateral and bilateral pCCND separately. In unilateral pCCND, patient with more than one metastatic node showed significantly higher recurrence than patients without nodal metastasis. In bilateral pCCND, the highest sensitivity/specificity was achieved in cutoff of $\geq 5$ metastatic lymph nodes. High MLN showed significant correlation on RFS in both unilateral and bilateral groups in univariate analysis, however, this correlation was no longer observed in multivariate analysis.

LNY was the total number of lymph node dissected during surgery, and it was used to assess the adequacy and completeness of node dissection. ${ }^{25)}$ Many researchers tried to find optimal node yield in PTC, however, there was no relevant cutoff point for minimum value of node to be dissected, especially in
pCCND. ${ }^{25,29)}$ Similarly, we hypophesis that high LNY might reduce local recurrences. For accurate analysis, we divided pCCND into unilateral and bilateral and set the each cutoff of LNY by ROC curve. LNY cutoff was 6 in unilateral PCCND and 11 in bilateral pCCND, but low/ high LNY did not show a statistical difference in RFS. It might because the more advanced the primary tumor was, the surgeon performed node dissection more completely. In fact, LNY showed positively associated trend with MLN in this study (Data no shown). Additionally, LNY could be influenced by not only surgeon factor but also by pathologic examination and combined disease such as thyroiditis. ${ }^{25)}$ Lymphadenopathy is common in autoimmune thyroiditis, it leads surgeon to perform more extensive nodal dissection when thyroiditis is accompanied with PTC. ${ }^{30)}$ A total $423(24.1 \%)$ showed combined autoimmune thyroiditis, of these 195 (34.9\%) patients was bilateral pCCND groups. Thyroiditis showed a significant correlation with LNY and LNR (data not shown), but thyroiditis itself did not show a significant correlation on RFS in both unilateral and bilateral pCCND.

Regarding nodal factors, it is noteworthy that only LNR of nodal factors remains an independent variable in multivariate analysis. LNR was the ratio of numbers of proven metastatic node to dissected total lymph node (MLN/LNY). LNR has recently attracted the spotlight because it represented surgery quality in terms of adequacy of nodal dissection (LNY) and quantitative aspect in terms of the amount of metastatic lymph nodes. ${ }^{27)}$ A large number of MLN or a small number of dissected nodes result in a high LNR. In previous reports, the cutoff of LNR varied between 0.11-0.86. ${ }^{8,24-27)}$ These discrepancies might arise because of different surgical intent (prophylactic versus therapeutic nodal dissection), different extent of lymphadenectomy (central compartment versus lateral neck area) and different nodal status (occult versus clinical metastasis). In this study, cutoff of LNR was $0.15 \leq$ LNR in unilateral pCCND and $0.3 \leq$ LNR in bilateral pCCND, and the reason of relatively lower cutoff of LNR than previous reports might be because the study population was limited to pCCND group in clinically NO patients. ${ }^{4,24-26)}$ 
Nodal Factors Impact on PTC with Prophylactic CCND

This study has several limitations. First, it is retrospective study of single surgeon's experiences in a single tertiary institution. However, on the other hand, single-surgeon, single-institution nature of this study could limit the bias associated with surgeon and pathologist factors. Second, even though this is large scaled study, the numbers of recurrent events are relative small to assess optimal cutoff and correlation of nodal factor on RFS. A multicenter study with high volume center will increase the sample size and achieve statistical significance of various nodal factors.

In conclusion, in regarding nodal factors including MLN, LNY and LNR, high LNR was only independent risk factor to worse RFS in both unilateral and bilateral PCCND in cNO PTC patients.

\section{Conflict of Interest}

The authors declare no conflict of interest.

\section{References}

1) Smith PW, Hanks LR, Salomone LJ, Hanks JB. Thyroid. In: Townsend CM, Beauchamp RD, Evers BM, Mattox KL, editors. Sabiston textbook of surgery: The biological basis of modern surgical practice. 20th ed. Philadelphia, PA: Elsevier; 2017. p.881-992.

2) Shigematsu N, Takami H, Ito N, Kubo A. Nationwide survey on the treatment policy for well-differentiated thyroid cancer -results of a questionnaire distributed at the 37th meeting of the Japanese Society of Thyroid Surgery. Endocr J 2005;52(4): 479-91.

3) American Thyroid Association Surgery Working Group, American Association of Endocrine Surgeons, American Academy of Otolaryngology-Head and Neck Surgery, American Head and Neck Society, Carty SE, Cooper DS, et al. Consensus statement on the terminology and classification of central neck dissection for thyroid cancer. Thyroid 2009;19(11): $1153-8$.

4) Haugen BR, Alexander EK, Bible KC, Doherty GM, Mandel SJ, Nikiforov YE, et al. 2015 American Thyroid Association management guidelines for adult patients with thyroid nodules and differentiated thyroid cancer: The American Thyroid Association guidelines task force on thyroid nodules and differentiated thyroid cancer. Thyroid 2016;26(1):1-133.

5) Barczynski M, Konturek A, Stopa M, Nowak W. Prophylactic central neck dissection for papillary thyroid cancer. Br J Surg 2013;100(3):410-8.

6) Amit M, Tam S, Boonsripitayanon M, Cabanillas ME, Busaidy NL, Grubbs EG, et al. Association of lymph node density with survival of patients with papillary thyroid cancer.
JAMA Otolaryngol Head Neck Surg 2018;144(2):108-14.

7) Kim Y, Roh JL, Gong G, Cho KJ, Choi SH, Nam SY, et al. Risk factors for lateral neck recurrence of N0/N1a papillary thyroid cancer. Ann Surg Oncol 2017;24(12):3609-16.

8) Nam SH, Roh JL, Gong G, Cho KJ, Choi SH, Nam SY, et al. Nodal factors predictive of recurrence after thyroidectomy and neck dissection for papillary thyroid carcinoma. Thyroid 2018;28(1):88-95.

9) Heaton CM, Chang JL, Orloff LA. Prognostic implications of lymph node yield in central and lateral neck dissections for well-differentiated papillary thyroid carcinoma. Thyroid 2016;26(3):434-40.

10) Popadich A, Levin O, Lee JC, Smooke-Praw S, Ro K, Fazel $\mathrm{M}$, et al. A multicenter cohort study of total thyroidectomy and routine central lymph node dissection for cNO papillary thyroid cancer. Surgery 2011;150(6):1048-57.

11) Hartl DM, Mamelle E, Borget I, Leboulleux S, Mirghani H, Schlumberger M. Influence of prophylactic neck dissection on rate of retreatment for papillary thyroid carcinoma. World J Surg 2013;37(8):1951-8.

12) Leboulleux S, Rubino C, Baudin E, Caillou B, Hartl DM, Bidart JM, et al. Prognostic factors for persistent or recurrent disease of papillary thyroid carcinoma with neck lymph node metastases and/or tumor extension beyond the thyroid capsule at initial diagnosis. J Clin Endocrinol Metab 2005;90(10):5723-9.

13) Podnos YD, Smith D, Wagman LD, Ellenhorn JD. The implication of lymph node metastasis on survival in patients with well-differentiated thyroid cancer. Am Surg 2005;71(9):731-4.

14) American Thyroid Association (ATA) Guidelines Taskforce on Thyroid Nodules and Differentiated Thyroid Cancer, Cooper DS, Doherty GM, Haugen BR, Kloos RT, Lee LS, et al. Revised American Thyroid Association management guidelines for patients with thyroid nodules and differentiated thyroid cancer. Thyroid 2009;19(11):1167-214.

15) Enyioha C, Roman SA, Sosa JA. Central lymph node dissection in patients with papillary thyroid cancer: A population level analysis of 14,257 cases. Am J Surg 2013;205(6):655-61.

16) Grodski S, Cornford L, Sywak M, Sidhu S, Delbridge L. Routine level VI lymph node dissection for papillary thyroid cancer: surgical technique. ANZ J Surg 2007;77(4):203-8.

17) Moo TA, McGill J, Allendorf J, Lee J, Fahey T 3rd, Zarnegar R. Impact of prophylactic central neck lymph node dissection on early recurrence in papillary thyroid carcinoma. World J Surg 2010;34(6):1187-91.

18) Kouvaraki MA, Shapiro SE, Fornage BD, Edeiken-Monro BS, Sherman SI, Vassilopoulou-Sellin R, et al. Role of preoperative ultrasonography in the surgical management of patients with thyroid cancer. Surgery 2003;134(6):946-54; discussion 54-5.

19) Roh JL, Kim JM, Park CI. Central lymph node metastasis of unilateral papillary thyroid carcinoma: patterns and factors predictive of nodal metastasis, morbidity, and recurrence. Ann Surg Oncol 2011;18(8):2245-50.

20) Roh JL, Park JY, Park CI. Total thyroidectomy plus neck dissection in differentiated papillary thyroid carcinoma patients: Pattern of nodal metastasis, morbidity, recurrence, and postoperative levels of serum parathyroid hormone. Ann Surg 
2007;245(4):604-10.

21) Sywak M, Cornford L, Roach P, Stalberg P, Sidhu S, Delbridge L. Routine ipsilateral level VI lymphadenectomy reduces postoperative thyroglobulin levels in papillary thyroid cancer. Surgery 2006;140(6):1000-5; discussion 5-7.

22) Ito $Y$, Miyauchi $A$, Masuoka $H$, Fukushima M, Kihara $M$, Miya A. Excellent prognosis of central lymph node recurrence-free survival for cNOMO papillary thyroid carcinoma patients who underwent routine prophylactic central node dissection. World J Surg 2018;42(8):2462-8.

23) Zheng CM, Ji YB, Song CM, Ge MH, Tae K. Number of metastatic lymph nodes and ratio of metastatic lymph nodes to total number of retrieved lymph nodes are risk factors for recurrence in patients with clinically node negative papillary thyroid carcinoma. Clin Exp Otorhinolaryngol 2018;11(1):58-64.

24) Park YM, Wang SG, Lee JC, Shin DH, Kim IJ, Son SM, et al. Metastatic lymph node status in the central compartment of papillary thyroid carcinoma: A prognostic factor of locoregional recurrence. Head Neck 2016;38 Suppl 1:E1172-6.

25) Vas Nunes JH, Clark JR, Gao K, Chua E, Campbell P, Niles $\mathrm{N}$, et al. Prognostic implications of lymph node yield and lymph node ratio in papillary thyroid carcinoma. Thyroid 2013;23(7):
811-6.

26) Schneider DF, Mazeh H, Chen H, Sippel RS. Lymph node ratio predicts recurrence in papillary thyroid cancer. Oncologist 2013;18(2):157-62.

27) Schneider DF, Chen H, Sippel RS. Impact of lymph node ratio on survival in papillary thyroid cancer. Ann Surg Oncol 2013;20(6):1906-11.

28) Randolph GW, Duh QY, Heller KS, LiVolsi VA, Mandel SJ, Steward DL, et al. The prognostic significance of nodal metastases from papillary thyroid carcinoma can be stratified based on the size and number of metastatic lymph nodes, as well as the presence of extranodal extension. Thyroid 2012;22(11): 1144-52.

29) Beal SH, Chen SL, Schneider PD, Martinez SR. An evaluation of lymph node yield and lymph node ratio in well-differentiated thyroid carcinoma. Am Surg 2010;76(1): 28-32.

30) Lai V, Yen TW, Rose BT, Fareau GG, Misustin SM, Evans $\mathrm{DB}$, et al. The effect of thyroiditis on the yield of central compartment lymph nodes in patients with papillary thyroid cancer. Ann Surg Oncol 2015;22(13):4181-6. 